\title{
Neonatal Sonographic Diagnosis of Tuberous Sclerosis
}

\author{
Mahesh ${ }^{1}$, Rithi Melissa D Silva², Prajnha U P ${ }^{3}$ \\ ${ }^{1}$ Senior Resident, Department of Radio Diagnosis, ${ }^{2}$ Junior Resident, Department of Radio Diagnosis, ${ }^{3} \mathrm{~K}$ S Hegde Institute of \\ Medical Scienes, Deralakatte, Mangaluru, India
}

Corresponding author: Mahesh, Senior Resident, Department of Radio Diagnosis, Father Muller Medical College and Hospital, Kankanady, Mangaluru-575002, India

DOI: http://dx.doi.org/10.21276/ijcmsr.2019.4.2.9

How to cite this article: Mahesh, Rithi Melissa D Silva, Prajnha U P. Neonatal sonographic diagnosis of tuberous sclerosis. International Journal of Contemporary Medicine Surgery and Radiology. 2019;4(2):B40-B42.

\section{A B S T R A C T}

Introduction: Tuberous sclerosis is a rare autosomal dominant neurocutaneous syndrome characterized by the presence of benign congenital tumors in multiple organs.

Case report: We report a case of tuberous sclerosis detected in preterm neonate by neurosonography and echocardiography. The diagnosis is usually established on the basis of diagnostic criteria applied to physical or radiologic findings.

Conclusion: Tuberous sclerosis may be diagnosed by ultrasonography in neonatal period if antenatal detection is missed by the presence of two of the following: cardiac rhabdomyomas, cortical tubers, subependymal nodules, and renal angiomyolipomas.

Keywords: Tuberous Sclerosis, Rhadomyoma, Tubers, Neurosonogram

\section{INTRODUCTION}

Tuberous sclerosis is a rare autosomal dominant neurocutaneous syndrome characterized by the presence of benign congenital tumours in multiple organs. The diagnosis is usually established on the basis of diagnostic criteria applied to physical or radiologic findings. ${ }^{1}$

Tuberous sclerosis is usually diagnosed in infancy or early childhood because a child presents with seizures, developmental delay, or hypomelanotic macules. However, the diagnosis of tuberous sclerosis can be made earlier or later on the basis of other features that manifest themselves at other ages. Cortical tubers and cardiac rhabdomyomas are one of the few findings detected prenatally and in infancy, whereas renal, pulmonary, and osseous lesions are identified more commonly in adulthood. Gomez developed a set of diagnostic criteria in 1998. These criteria were sorted by major and minor features, and a definite diagnosis was made when two major features or one major and two minor features were present. A probable diagnosis was made when one major and one minor feature were present, and a possible diagnosis when one major or two or more minor features were present. $^{2}$

In our case report we are presenting a case of antenatally detected cardiac anomaly which in postnatal period was diagnosed to be one of the major criteria for tuberous sclerosis.

\section{CASE REPORT}

A preterm (33 weeks) male baby, with appropriate growth for gestational age was delivered by normal vaginal delivery to a 20-year-old female on 16/06/2018 at 2:03 pm. The birth

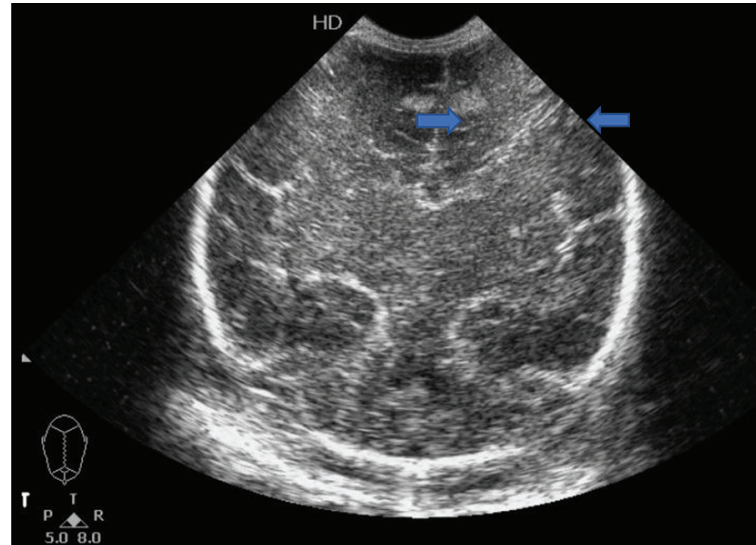

Figure-1: Coronal section, Arrow marks showing hyperechoic cortical tubers

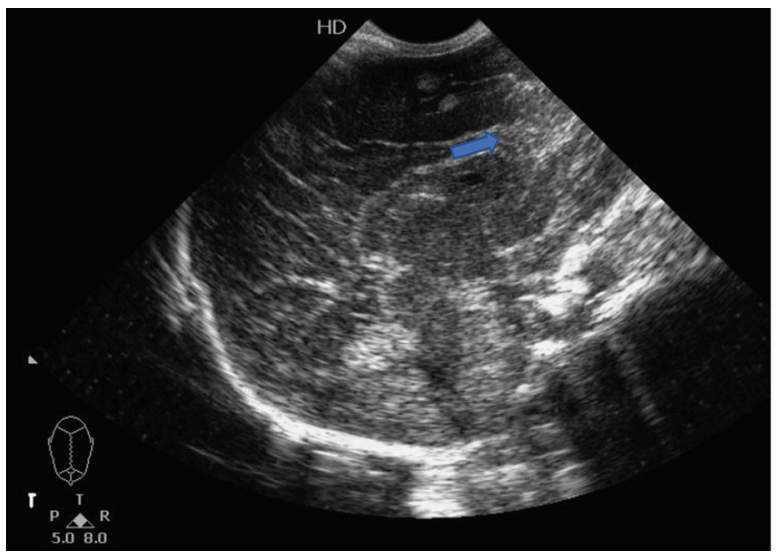

Figure-2: Sagittal section, Arrow marks showing hyperechoic cortical tubers 


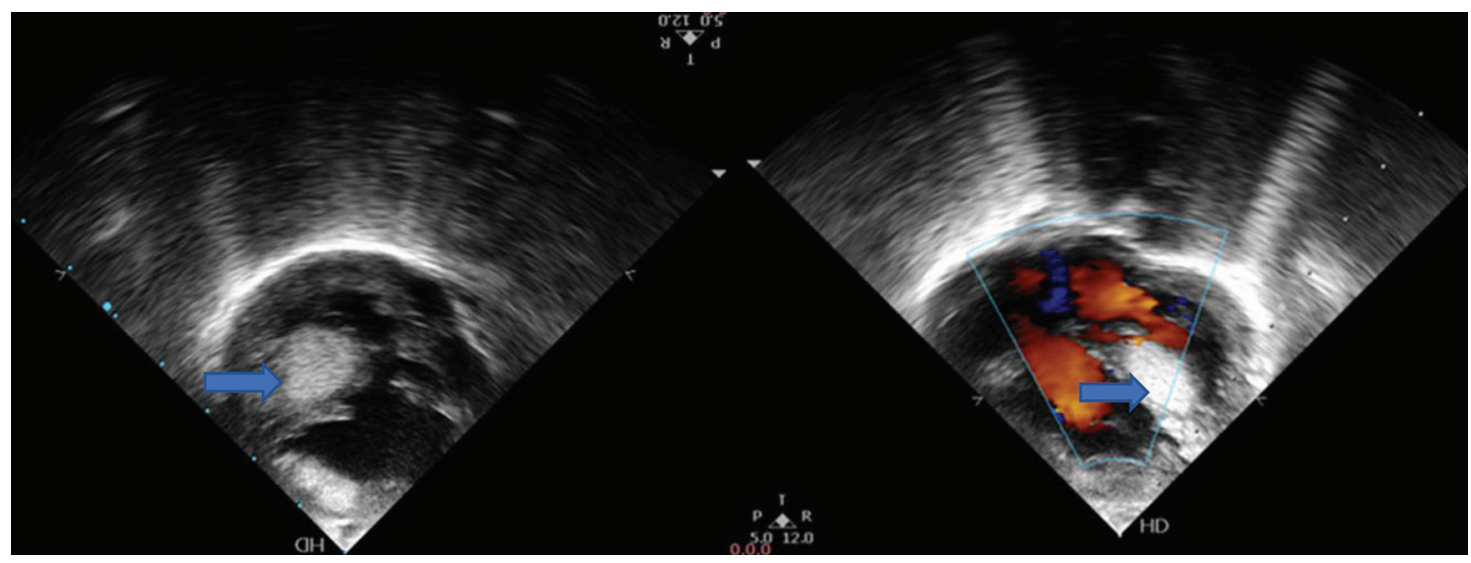

Figure-3: ECHO images, Arrow marks showing hyperechoic cardiac rhabdomyomas.

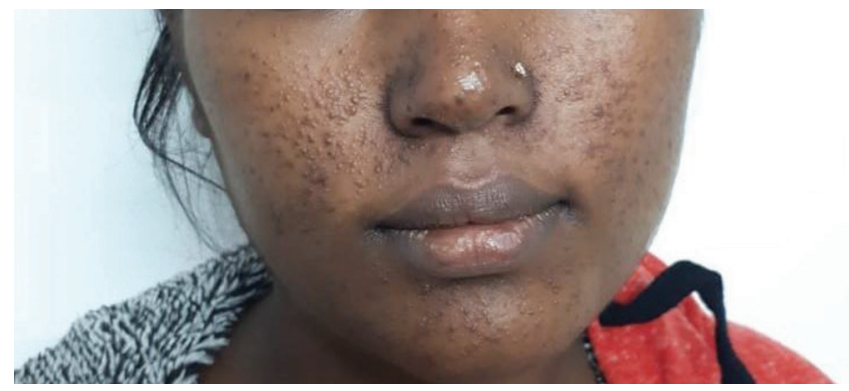

Figure-4: Adenoma sebaceum in the mother

weight was 1600 grams with an APGAR of 6 and 7 at 1 min and $5 \mathrm{~min}$ respectively. The baby did not cry at birth and was intubated and shifted to neonatal ICU. At admission, cry, tone and activity was poor. The baby was tachypnoeic not maintaining saturation at room air. Head to toe examination revealed dysmorphic facies, metopic suture, severe subcostal and intercostal retraction, undescended left testes and flat feet. Sepsis screen was negative on day 0 and blood gas showed respiratory acidosis. Surfactant was given and baby was connected to ventilator.

An antenatal scan at 30 weeks showed foetal hypoplastic left ventricle, double outlet right ventricle and VSD.

On day 2, baby had 1 episode of convulsion and was started on anticonvulsants. Neuro-sonogram, ultrasound abdomen and Echo were advised. Neuro-sonogram revealed iso to hyperechoic foci in cortical gyri of bilateral frontal, temporal and parietal lobes - consistent with cortical tubers (figure 1,2). There was no evidence of subependymal nodules or subependymal giant cell astrocytoma.

Echocardiography revealed double outlet right ventricle, large mal-aligned VSD with bidirectional shunt, small ASD, Pulmonary arterial hypertension, and right ventricular dysfunction. Multiple hyperechoic intracardiac masses were seen in interventricular septum and right ventricle free wall which were likely to be rhabdomyomas (figure-3).

Ultrasound abdomen revealed oedematous gall bladder wall with small pericholecystic collection and minimal ascites.

With the evidence of above imaging findings, diagnosis of tuberous sclerosis was made.

Retrospectively, maternal examination revealed multiple, discrete, reddish brown papules distributed symmetrically over malar region involving nasolabial fold suggestive of adenoma sebaceum (figure-4).
Repeat sepsis screen on day 2 showed increasing trend of CRP. Baby had persistent metabolic acidosis and developed acute kidney injury.

On day 5, baby had bloody nasogastric aspirate and electrolyte imbalance and was treated accordingly. Baby continued to have persistent desaturation and bradycardia. Cardiopulmonary resuscitation was initiated and despite of all resuscitative measures baby could not be revived and was declared dead .

\section{DISCUSSION}

TSC is characterized by the growth of benign hamartomas in the brain, the kidneys, the heart, and the skin. During the antenatal cardiac rhabdomyomas are often the first manifestation which can be diagnosed by ultrasonograhy and MRI. However, it remains challenging to detect hamartomas in other organs such as the brain, the kidneys, the heart, and the skin. ${ }^{4}$

Our study demonstrated that it is feasible to make a diagnosis of Tuberous sclerosis complex (TSC) by an integrated approach of clinical examination and ultrasonography in the neonatal period.

Revised clinical diagnostic criteria for tuberous sclerosis includes major and minor criteria. ${ }^{3}$

Major features being:

1. Hypomelanotic macules ( $\geq 3$, at least $5-\mathrm{mm}$ diameter)

2. Angiofibromas $(\geq 3)$ or fibrous cephalic plaque

3. Ungual fibromas $(\geq 2)$

4. Shagreen patch

5. Multiple retinal hamartomas

6. Cortical dysplasias (Includes tubers and cerebral white matter radial migration lines)

7. Subependymal nodules

8. Subependymal giant cell astrocytoma

9. Cardiac rhabdomyoma

10. Lymphangioleiomyomatosis (LAM)

11. Angiomyolipomas $(\geq 2)$

(A combination of the two major clinical features (LAM and angiomyolipomas) without other features does not meet criteria for a definite diagnosis)

Minor features being:

1. "Confetti" skin lesions

2. Dental enamel pits $(>3)$

3. Intraoral fibromas $(\geq 2)$ 
4. Retinal achromic patch

5. Multiple renal cysts

6. Nonrenal hamartomas

Definite diagnosis: Two major features or one major feature with $\geq 2$ minor features

Possible diagnosis: Either one major feature or $\geq 2$ minor features

In our case, 2 major criterias were fulfilled and hence a diagnosis of tuberous sclerosis was made. Despite of all resuscitative measures, the neonate could not be revived after day 5 of birth.

The natural history of cardiac rhabdomyomas is for regression to occur in the first years of life. Subependymal nodules and cortical tubers, while not the most common presenting sign, are a very frequent finding in infants receiving a TSC diagnosis. ${ }^{5}$

\section{CONCLUSION}

Pediatricians and pediatric subspecialists should be aware of prenatal and postnatal imaging findings relevant to TSC, as the presence of more than one finding is sufficient for TSC diagnosis. A suspicion of TSC based on an ultrasound finding of a cardiac, neurological, or renal mass should prompt further imaging.

\section{REFERENCES}

1. S. Umeoka, T. Koyama, Y. Miki, M. Akai, K. Tsutsui, K. Togashi. Pictorial review of tuberous sclerosis in various organs Radiographics 2008;28:e32.

2. Comprehensive Imaging Manifestations of Tuberous Sclerosis. Saro B. Manoukian1 Daniel J. Kowal. AJR 2015; 204:933-943.

3. Northrup H, Krueger DA, International Tuberous Sclerosis Complex Consensus Group. Tuberous sclerosis complex diagnostic criteria update: recommendations of the 2012 Iinternational Tuberous Sclerosis Complex Consensus Conference. Pediatr Neurol. 2013;49:243254.

4. Antenatal screening and diagnosis of tuberous sclerosis complex by fetal echocardiography and targeted genomic sequencing. Xiaoyan Gu et al. Medicine 2018; 97:15-20.

5. Multimodal Imaging in the Prenatal Diagnosis of TuberousSclerosisComplex.Gusman M, Servaes S, Feygin T, Degenhardt K, Epelman M. AJR 2000;175:1067-1069.

Source of Support: Nil; Conflict of Interest: None

Submitted: 04-04-2019; Accepted: 25-04-2019; Published online: 19-05-2019 